\title{
DEVELOPMENT OF A CARBURIZING AND QUENCHING SIMULATION TOOL: DETERMINATION OF HEAT TRANSFER BOUNDARY CONDITIONS IN SALT
}

\author{
Douglas Shick \\ The Torrington Company, Torrington, Connecticut \\ Donald R. Chenoweth \\ Sandia National Laboratory, Livermore California \\ Nagendra Palle, Claude Mack, Warren Copple \\ Ford Motor Co.. Dearborn Michigan \\ Wen-Tzong Lee \\ J. P. Industrial, Inc., Ann Arbor Michigan \\ William Elliott, James Park, Gerald M. Ludtka, Roberto Lenarduzzi \\ Oak Ridge National Laboratory, Oak Ridge Tennessee \\ Harry Walton \\ The Torrington Company, Rutherfordton, North Carolina \\ Maurice Howes \\ IITRIINFAC, Chicago, Illinois
}

CONF- $961140--3$

\begin{abstract}
In the numerical simulation of the quenching of steel parts. it is desirable to limit the thermal problem to one of conduction in the solid part. with Tewtonian cooling on the surface. This avoids the solution of highly transient. non-Boussinesq conjugate heat transfer problems which ofien involve mixed convection. Of course. the heat transfer coefficient $h$ is in general a function of local surface temperature. and may depend strongly on the part geometry and other parameters. In order to obtain appropriate heat transfer coefficients for quenching thick rings in salt. we solve the conjugate heat transfer problem for representative geometries and determine the heat transfer coefficient from the calculated heat flux at the solid-fluid interface. This is done in a two-stage approach, First. we examine fully coupled solutions obtained from first principles without adjustable empirical parameters using an adaprive finite difference code applicable to simple shapes. The purpose of this part of the procedure is to gain insight into the physics of immersion. free convection and forced convection and to determine the most appropriate forms of the physical properies in the salt. Validation for simple shapes is accomplished by comparison with experimental data reported in the literature. Second. empirical modifications to physical properties and simplified governing equations are used to account for conduction-dominated heat transfer on initial contact and subsequent forced convection during immersion, based on results of the first calculations. so that a commercial finite-volume CFD code can be used to model complex shapes and now in a quench tank. These results are compared with experimental data for thick rings quenched in salt. and used to calculate surface heat transfer coefficients as functions of temperature and location from the resufting heat flux at the part surface.
\end{abstract}

IT IS COMMON IN SOLID-FLUID heat transfer calculations to locally apply constant or surface-averaged heat-transfer coefficients obtained from either free or forced steads convection over simple shapes with small temperature differences from the ambient fluid. This procedure avoids the solution of highly transient. non-Boussinesq conjugate heat transfer problems which often involve mixed convection. but it is not generally adequate to represent the large temperature differences and complicated flow that occurs in typical immersion heat treatment applications Still, it is desirable in the numerical simulation of the quenching of sieel parts to limit the thermal par of the problem to one of conduction in the solid part. with Neutonian cooling on the surface, so the thermal boundary condition has the form

$$
q=h_{;} A\left(T_{1}-T_{0}\right) \text {. }
$$

In this paper. we demonstrate for small pars (dimensions on the order of inches rather than feet) quenched in molten salt that it is feasible to calculate non-uniform surface heat transfer from first principles without adjustable empirical parameters. We use literature physical property salt data (15) for $T<1000^{\circ} \mathrm{F}$. and then extrapolate it to the initial part temperature. The reported thermal/chemical breakdown of NaNO: for $T>800^{\circ} \mathrm{F}$ is not considered to be important due to the shor time the surface temperature exceeds that value for small parts. Similarly, for small parts. the local Reynolds and Rayleigh numbers are below the corresponding critical values for most if not all of the quench. so we see no evidence of significant turbulence effects. only some large scale unsteadiness for brief periods.

Motivated by the above results. empirical modifications to physical properties and simplified governing equations are used to account for conductiondominated heat transfer on initial contact with the quenchant and subsequent forced convection during immersion. so that a commercial FE code can be used to model complex shapes and flow in a quench tank. These results are compared with experimental data for thick rings quenched in salt, and used to calculate surface heat transfer 


\section{DISCLAIMER}

Portions of this document may be illegible in electronic image products. Images are produced from the best available original document. 

Feturn to this estimate of $C$, following the discussion of thermal diffusivily and thermal conductivity.

\section{Thermal Diffusivity}

The validity of the procedure just mentioned is difficult to prove since it is the $T$ dependence of $k$ and $a$ which has been the most difficult to confirm. Both large positive and negative $T$ slopes have been reponed in the literature since 1952. In some cases investigators have in facl assumed constant heat capacity and then used $a=k / \rho C_{r}$ to infer either $k$ or $\alpha$ behavior from the measurements of the other. During the last two decades it has been reporied somewhat consistently that the thermal diffusivity increases with increasing $T$. while thermal conductivity appears to have a weak decrease with $T$. These small positive and negative slopes with $T$ for $\alpha$ and $k$, respectively, have been reported for fairly narrow ranges of temperature. Thus if extrapolated to the pant temperature. they can still represent significant effects.

Neither Kirst et al. nor Nissen repon any thermal diffusivity or conductivity data. Obviously both $a$ and $k$ are not needed for CFD if $\mu, \rho$ and $C_{\theta}$ are available. However information on both $\alpha$ and $k$ does allow a valuable intemal consistency check. especially with respect to temperature dependence.

Ohta et al. [10] show for $\mathrm{NaNO}_{1}$ that $\alpha$ increases from about 0.0014 to $0.0015 \mathrm{~cm}^{2} / \mathrm{sec}$ as $T$ increases from 593 to $660 \mathrm{~K}$. while for $\mathrm{KNO}_{3} \alpha$ increases from about 0.0015 to $0.0016 \mathrm{~cm}^{2} / \mathrm{sec}$ as $T$ increases from 621 to $694 \mathrm{~K}$. Some of the same authors appear to report data by the same experimental method on the same materials in Ref. [11]. but with significantly higher magnitudes. 0.001910 $0.0021 \mathrm{~cm}^{2} / \mathrm{sec}$ for both $\mathrm{KNO}_{2}$ and $\mathrm{NaNO}_{3}$. without explaining the apparent differences. Kato ef al. [12, 13] find that $\alpha$ is about constant for $\mathrm{KNO}$, at about 0.001694 $\mathrm{cm}^{2} / \mathrm{sec}$ and similarly for $\mathrm{NaNO}_{3}$ at about 0.001722 $\mathrm{cm}^{2} / \mathrm{sec}$. Odawara ef al. (14) also repon $\alpha$ for $\mathrm{NaNO}_{\text {, to be }}$ about constant for $581<T<691 \mathrm{~K}, \alpha=0.00169 \mathrm{~cm}^{2} / \mathrm{sec}$. but for LOTS they find that $\alpha$ increases from 0.00168 to $0.00181 \mathrm{~cm} / \mathrm{sec}$ as $T$ increases from 440 to $692 \mathrm{~K}$ :

$$
\alpha(\mathrm{cm} ; \mathrm{sec})=0.001[1.45+0.000516 T(\mathrm{~K})] \text {. }
$$

\section{Thermal Conductivity}

Odawara et al. $\left[14\right.$ \} also use constant $C_{\text {, for that }}$ temperature range, with density linearly dependent on $T$. and estimate $k=\alpha \rho C_{p}$ for LOTS to be

$$
k(W / m / K)=0.5385-0.000047 T(K) .
$$

so that had $C_{p}$ also decreased with $T$, the very weak $T$ slope of this $k$ would have been strengthened somewhat. Omotani et al. [15] used various mixtures of $\mathrm{KNO}_{3}$ and $\mathrm{NaNO}_{3}$ to establish that thermal conductivity appears to follow a LMMR. For the HOTS (equimolar mixture), a slightly negative $T$ dependence was obtained for $T$ between 498 and $593 \mathrm{~K}$

$$
k(\mathrm{~W} / \mathrm{m} / \mathrm{K})=0.5461-0.000169 T(\mathrm{~K}) .
$$

Omotani et al. [16] gave a comparable result for the LOTS (ternary mixture) for $427<T<584 \mathrm{~K}$. showing even weaker dependence on $T$ than for HOTS, similar to that of Odawara et al. [14].

$$
k(W / m / K)=0.5163-0.000038 T(K) \text {. }
$$

The results of Tufeu et al. [9] are in substantial agreement with Omotani et al. [16] for HOTS, however they obtained a slightly positive $T$ dependence for LOTS:

$$
k(W / m / K)=0.4541+0.00058 T(K) \text {. }
$$

Kitade er al. [17] give results for pure NaNO, and pure $\mathrm{KNO}_{3}$ in the narrow $T$ ranges $584<T<662 \mathrm{~K}$ and $622<T<$ $712 \mathrm{~K}$. respectively. If these results are combined with a LMMR for the equimolar case. for HOTS:

$$
k(W / m / K)=0.6239-0.000265 T(K) \text {. }
$$

More recently DiGuilio and Tegals] gave similar results for HOTS for $525<T<590 \mathrm{~K}$.

$$
k(W / m / K)=0.635-0.000317 T(K) \text {. }
$$

which is very similar to Eq. (11). We recommend and use Eq. (12) for HOTS (high operating temperature salts) for near equimolar mixures of $\mathrm{KNO}_{3}$ and $\mathrm{NaNO}_{3}$. For LOTS (low operating temperature salts) consisting of ternary mixtures similar to HTS (HITEC) we recommend and use Eq. (7) which Odawara et al. [14] estimated from their thermal diffusivity results via a least-squares fit. That expression has coefficients whose percentage increase over the lower values of Omotani et al. [15] have the same ratio 5.5 as those of DiGuilio and Teja[s] when compared to Omotani et al. results for HOTS. That is, we are assuming comparable systematic errors for both LOTS and HOTS in the results of Omotani et al. Therefore. for HOTS, $\alpha=0.0016 \mathrm{~cm}^{2} / \mathrm{sec}$, a constant. and

$$
C_{D}(\mathrm{cal} / \mathrm{g} / \mathrm{K})=\frac{0.419[1.0-0.0005 T(\mathrm{~K})]}{1.0-0.000281 T(\mathrm{~K})},
$$

which decreases about $5 \%$ as $T$ increases from 500 to 650 K. For LOTS. $C_{p}=k / \alpha \rho=0.376 \mathrm{cal} / g / \mathrm{K}$, a constant and

$$
\alpha\left(\mathrm{cm}^{2} / \mathrm{sec}\right)=0.001[1.45+0.000516 T(\mathrm{~K})]
$$

These give a fully consistent set of physical properties. considering the limited temperature range as well as the considerable scatter in much of the experimental results for $k$ and $a$.

\section{Thermal Decomposition of Quenchant Components}

It is well known[1] that LOTS undergoes a thermal decomposition above $700 \mathrm{~K}$. In this ternary mixture. it is the nitrite which is most unstable, breaking down to nitrate. oxide and nitrogen gas at a rate which increases with temperature. Fortunately, according to Bradshaw and Meeker. [18] the mixture adjacent to the part surface must remain above this breakdown temperarure for periods greater than about a minute, in order for enough decomposition to occur to significantly affect physical properies. Therefore the quenching of small parts is not

$$
\text { Shick }
$$


Ruid is well known: it has been studied for Covetle flow by Renardy, [19] and for Poiseuille Now by Pinarbasi and Liakopoulos.[20] It is sometimes referted to as the "thin layer effect" or as "lubrication stabilization" and it can greatly delay transition to turbulence. This same effect can also greatly alter mixed convective behavior. such that a flow which would be turbulent in free convection. can be parially laminarized by the additional influence of forced convection when it is added to the problem.(21.22) In fact Kitamura and inagaki[23] found that on a vertical heated flat plate with upuard forced water flow, the "turbulence suppression" is so great that the mixed convection heat. transfer coefficient or Nusselt number can be lowered by as much as $25 \%$ compared to that from either pure forced or pure free convection with comparable parameters.

\section{COMPUTATIONAL METHODS: COMPLETE ANALYSIS}

The computational methods utilized to predict the quenching behavior of stainless steel probes and vertical cylinders were developed during the late $1970^{\circ} \mathrm{s}$ and early: $1980 \mathrm{~s}$. They are embodied in a time-dependent, twodimensional code. TRACE2D. which solves the fully nonBoussinesq equations (variable physical properties) using direst Poisson solvers based on those of Adams et al. [24]

TRACE2D also utilizes an independent variable transformation to a non-physical computational plane. where the calculations are performed using uniform finitedifference meshes.[25.26] However in the physical plane. these grid points can have a highly non-uniform spatial distribution neas part surfaces which can be adapted towards a uniform distribution as time evolves. This is done using thermal gradients at the part surface to control the adaption process. and assure that these gradients are accurately resolved at eariy time where they are largest. This procedure has allowed TRACE2D to be modified to describe parts of simple shape (arbitrary aspect ratio cylinders and rings) which are placed in motion vertically to simulate actual immersion processes.

TRACE2D has also been generalized to include conjugate heal transfer between finite solid parts and a surrounding liquid quenchant. Thus the heat-transfer process between the part. where conduction is dominant. and the quenchant, where mixed convection (including arbitrary relative amounts of free and forced convection) may be present, is fully coupled. There are no simplifying assumptions other than acoustic wave filtering in the quenchant flow equations. That is, the compressible Navier-Stokes equations which are solved by TRACE2D are simplified only by the small Mach number approximation. This allows the time-step limitation for numerical stability of explicit finite-difference methods to be greatly enlarged. This is accomplished without any appreciable penalty provided the quenchant velocity squared is always much smaller than the local sound speed squared.

The entire procedure was fully validated using air in the free convection regime by Chenoweth and Paolucei[27, 28] for laminar flow, where the Rayleigh number Ra remained less than $10^{\prime}$. It was also validated in the transition region where $10^{*}<\mathrm{Ra}<10^{\prime \prime}$, during which unsteady periodic motion progressed toward fully chaotic motion as urbulen motion was approached [29] Finally. TRACE2D u.as used to predict the effects of fully turbulent motion 30 ] where $R a$ $=10^{\prime \prime}$. This validation process is important because it is found that comparable Rayleigh numbers are encouniered here, but usually in the mixed convection region where the Reynolds number also plays a key role.

In the results discussed in the next section. the temperature dependent physical properties for mild steel and stainless sieel were obtained from Bogaard and Desai[31] and Touloukain and Ho, 32\} respectively. The physical properties used for salt were those discussed above.
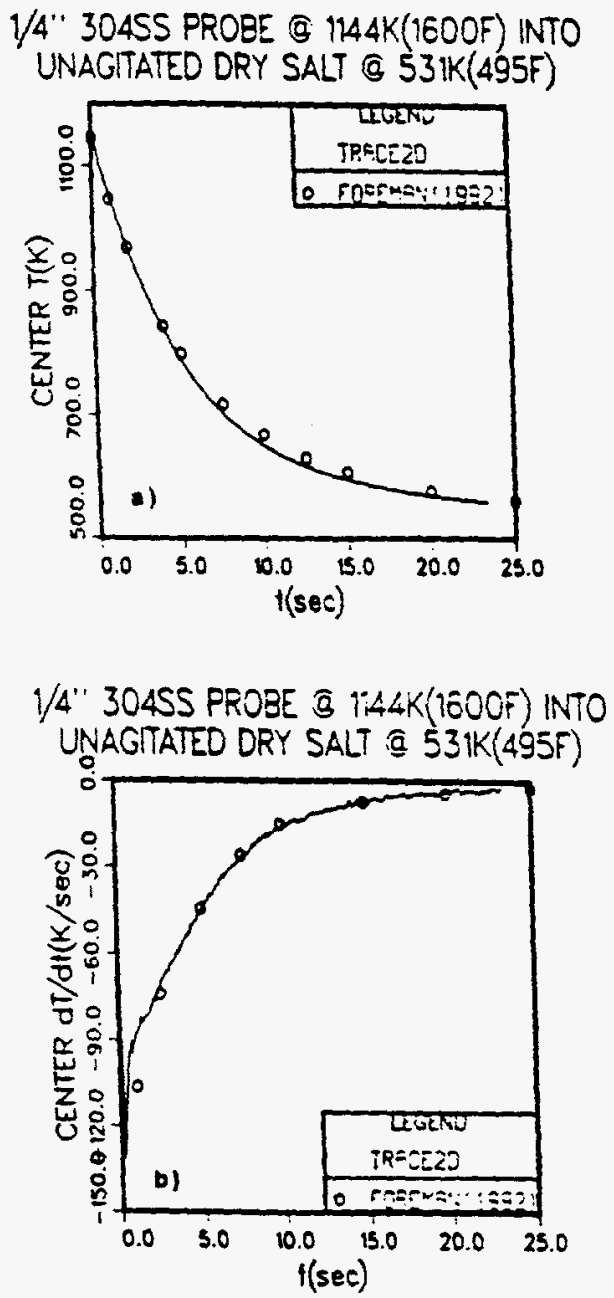

Figure 1. Comparison of predicted a) center temperature and b) cooling rate for $1 / 4$-inch-diam stainiess steel probe quenched in dry salt with experimental data of Foreman [33].

\section{Results and Discussion}

Foreman[33] gave the center temperature and cooling rate of a 0.25 -inch-diameter 304 stainiess steel probe initially at $1600^{\circ} \mathrm{F}$ (1144 $\mathrm{K}$ ) quenched into unagitated dry salt (HOTS) at $495^{\circ} \mathrm{F}(531 \mathrm{~K})$. Such a small diameter probe can be expected to exhibit a cooling rate which is initially 
parts being quenched, the quench tank geometry, and forced flow or agitation of the quenchant in the tank.

In the previous sections. we have demonstrated for small parts quenched in molten salt that it is feasible to calculate non-uniform surface heal transfer from first principles without adjustable empirical parameters. For small parts. the local Reynolds and Rayleigh numbers are below the corresponding critical values for most if not all of the quench. so that we see no evidence of the existence of significant turbulence effects. However, it is stull not practical to perform a large-scale simulation such as we require in this way.

HOWES ONE INCH SS CYIINDER

(9 1773K(1652F) INTO SALT @ 473K(392F)

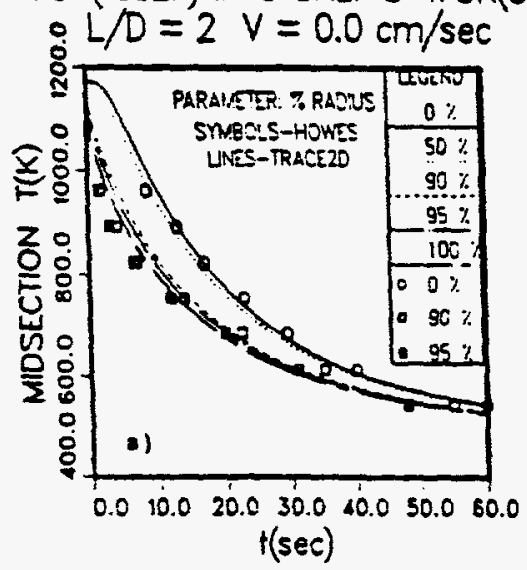

HOWES ONE INCH SS CYLINDER (3) 173K(1652F) INTO SALT $\hat{a}$ 473K(392F)

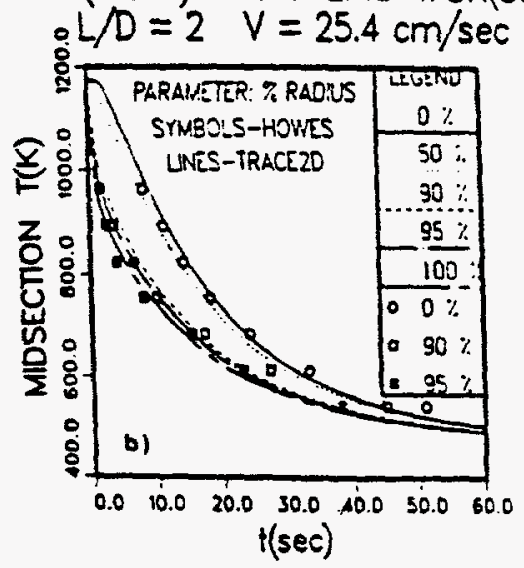

Figure 4. Comparison of predicted mid-section temperature for 1-inch-diam stainless steel cylinder quenched in dry salt with experimental data of Howes [35].

A typical cooling curve showing surface temperature of a thick ring quenched in salt is shown in Figure 6. From these cooling curves. and from those shown for cylindrical probes in Figs. 1-5, we can identify two distinct stages that the par experiences during the quench:
HOWES TWO INCH SS CYLINDER

(a) 173K(1652F) INTO SALT @ $473 \mathrm{~K}(392 F)$

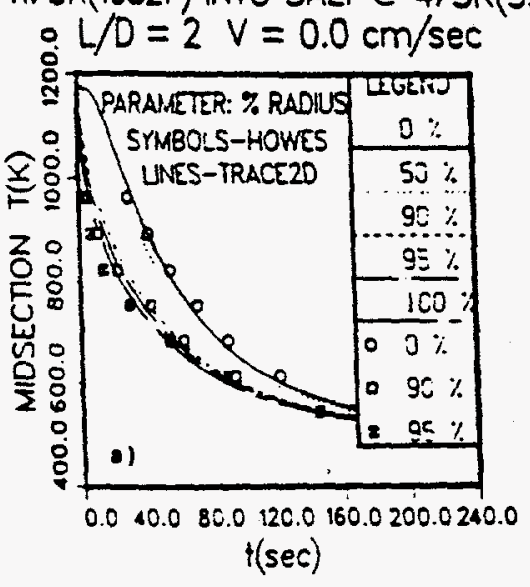

HOWES THREE INCH SS CYLINDER

(a) 173K(1652F) INTO SALT @. $473 \mathrm{~K}(392 \mathrm{~F})$

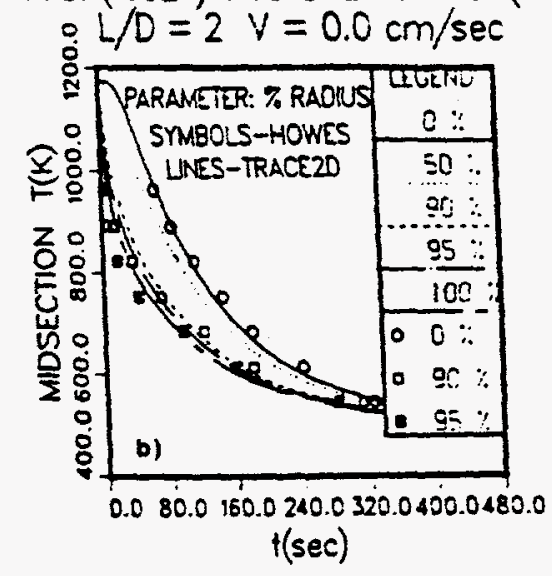

Figure 5. Comparison of predicted mid-section temperature for 2- and 3-inch-diam stainless steel cylinder quenched in dry salt with experimental data of Howes [35].

- Enhanced thermal transpor during immersion (conduction-dominated initial contact, giving uay 10 forced and natural convection as the part descends):

- Cooling due to natural convection once the part has reached maximum depth.

From the discussions above, and in particular Eq. (16). it can be shown that the thermal boundary layer in the salt adjacent to the part is on the ordes of $0.1 \mathrm{~mm}$ during the initial stages of the quench. To accurately capture the thermal gradient in a numerical simulation. this boundary layer would have to be resolved which requires the discretization to be of the same length scale. Unfortunately, such a fine grid or mesh is impractical in large scale simulations. It is advantageous. from the standpoint of computational efficiency. to make some simplifying assumptions in the problem formulation. We will account explicitly only for natural convection and 


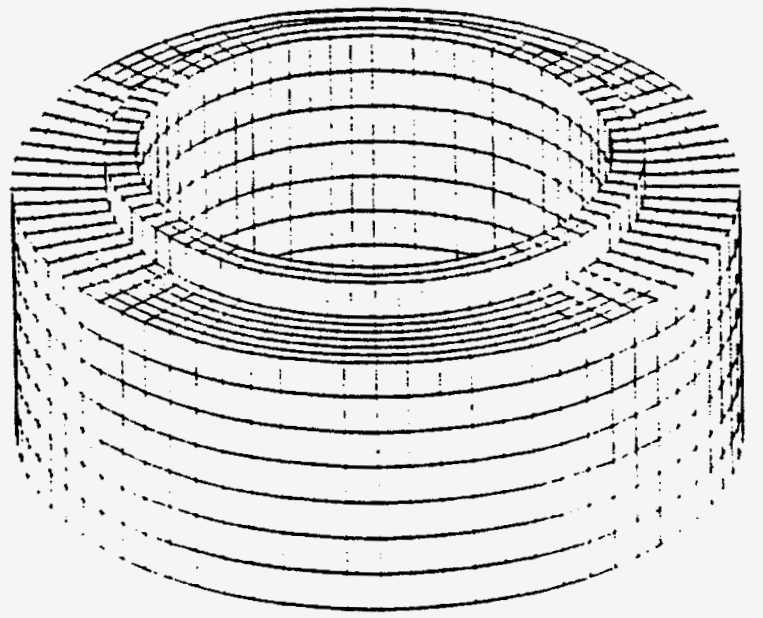

Figure 9. Finite volume STAR.CD mesh of ring.

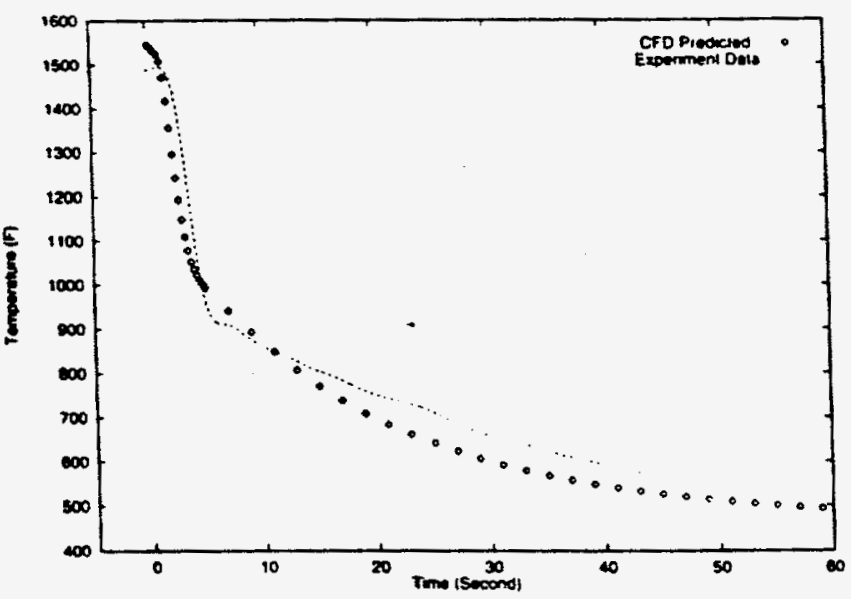

Figure 10. Calculated and experimental cooling curves showing surface temperature of the thick ring.

\section{ACKNOWLEDGEMENT}

This effort was sponsored by the Manufacturing Technology Directorate, Wtight Laboratory (WL/MTX). Air Force Material Command, USAF, under cooperative agreement award F33615-96-5619 to the National Center for Manufacturing Sciences. Inc. The U.S. Government is authorized to reproduce and distribute reprints for Governmental purposes notwithstanding any copyright notation thereon. The views and conclusions contained herein are those of the authors and should not be interpreted as necessarily representing the official policies or endorsements, either expressed or implied, of Wright Laboratory or the U.S. Government.

Research sponsored by the U.S. Department of Energy, Defiense Programs, Assistant Secretary, Technology Management Group, Technology Transfer Initiative under contract number DE-AC05-96OR22464 with Lockheed Martin Energy Research Corporation.

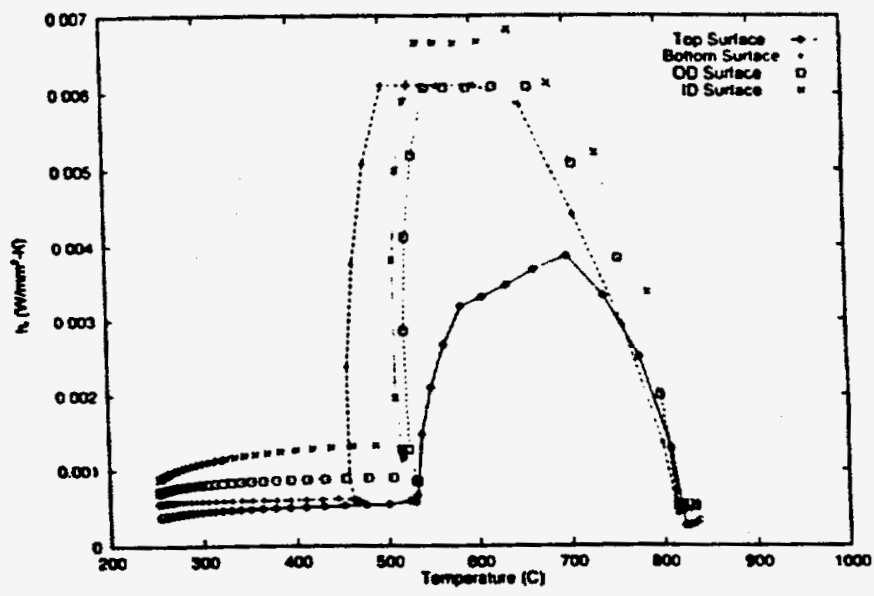

Figure 11. Heat transfer coefficients derived from the calculated surface heat flux.

\section{REFERENCES}

1. W.E. Kirst, W.M. Nagle. and J.B. Castner. Trans. Am. Inst. Chem. Eng. 36. 371 (1940).

2. D.A. Nissen, "The Chemistry of the Binary NaNO-KNO System," SAND81-8007, Sandia Nat. Lab.. Livermore CA (June. 1981).

3. D.A. Nissen. J. Chem. Eng. Data 27. 269-273 (1982).

4. R.W. Carling. Thermochim. ACTA 60, 265 (1983).

5. R.M. DiGuilio and A.S. Teja. Int. J. Thermophys. 13. 575-592 (1992).

6. G.J. Janz, C.B. Allen. N.P. Bansal, R.M. Murphy. and R.P.T. Tomkins. Physical Properties Data Compilations Relevent to Energy Storage. Part II Molten Salts: Data on Single and Multi-Component Salt Systems (NSRDS-NBS 61. April 1979).

7. Y. Takahashi, Sakamoto. R., and Kamimoto. M. Int. J. Thermophys. 9, 1081-1090 (1988).

8. GJ. Janz and G.N. Truong. J. Chem. Eng. Data 28. 201 (1983).

9. R. Tufeu, J.P. Petitet, L. Denielou. and B. Le Neindre. Int. J. Thermophys. 6, 315-330 (1985).

10. H. Ohta, G. Ogura, Y. Waseda, and M. Suzuki, Rer. Sci Instrum. 61, 2645-2649 (1990).

11. Y. Waseda. M. Masuda. and H. Ohta, in Proceedings of the Fourth Int. Symposium on Advanced Nuclear Energy

Res., Feb 5-7, 1992 (Mito, Wraki. Japan, 1992). 298-301

12. Y. Kato. K. Furukawa, N. Araki, and K. Kobayasi, $8 t h$ ETPC Proceedings 15, 73-80 (1983).

13. Y. Kato, K. Furukawa. N. Araki, and K. Kobayasi. High Temperatures-High Pressures 15, 191-198 (1983).

14. O. Odawara, I. Okada. and K. Kawamura, J. Chem. Eng. Data 22, 222-225 (1977).

15. T. Omotani, Y. Nagasaka, and A. Nagashima, Int. J. Thermophys. 3, 17-25 (1982).

16. T. Omotani and A. Nagashima, J. Chem. Eng. Data 29. 1-3 (1984).

17. S. Kitade, Y. Kobayashi, Y. Nagasaka, and A.

Nagashima. High Temperarures-High Pressures 21.219-

224 (1989). 